LA-UR $35=568$

TITLE:

AUTHOR(S):

SUBMITED TO:
A NEW METHOD OF DETERMINING THE EJECTED MASS OF NOVAE

Warren M. Sparks, X-3

Padova-Abano Conference on Cataclysmic Variables: Inter Class Relations Abano, Italy

June 19-25, 1994

$\cdot$

Los Alamos

DISTRIBUTION DE. IHIS DOCUMENT. IS UNLIMITE $\mathcal{N}$ (P)

Los Alamos National Laboratory Los Alamos, New Mexico 87545

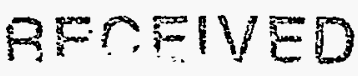




\section{DISCLAIMER}

Portions of this document may be illegible in electronic image products. Images are produced from the best available original document. 


\title{
A NEW METHOD OF DETERMINING THE EJECTED MASS OF NOVAE
}

\author{
WARREN M. SPARKS \\ Los Alamos National Laboratory \\ MS F663 \\ Los Alamos, NM 87544 \\ USA
}

\begin{abstract}
.
A new method of determining the ejected mass of novae based on simple, reasonable assumptions is presented. This method assumes that the remnant mass on the white dwarf is the same as that from the previous nova outburst. The hydrogen, helium, and metal abundances of the accreted material from the secondary must also be known or assumed. The white dwarf's mass has a small effect because the amount of hydrogen consumed during the thermonuclear runaway only depends weakly upon this mass. If the composition of the ejecta and the time of the remnant shell burnout are determined from observations, then the ejected and remnant masses can be deduced. At present only a sharp decrease in the $\mathrm{X}$-rays observed by ROSAT has been attributed to this remnant burnout and only for two novae: GQ Mus and V1974 Cyg. The ejected and remnant masses for these two novae are calculated. If other indicators of nova remnant burnout, such as a rapid decrease in high-ionization lines, can be identified, then this method could be applied to additional novae.
\end{abstract}

\section{Introduction}

The ejected mass is one of the most basic quantities in nova studies, but it has been very difficult to determine observationally. Most ejected mass determinations involve relating an electron temperature from the ratio of a forbidden line pair, the emission measurement from the flux of a hydrogen 
line, and an estimate of the ejecta's volume. The nonuniformity of the ejecta causes a large uncertainity in these mass determinations.

Nova ejecta consists of material from three sources: accreted material from the secondary, remnant material from the previous outburst, and dredged-up white dwarf material. These three sources are shown schematically as layers on the white dwarf in the first panel of the drawing in Figure 1. The next panels picture the mixing due to the thermonuclear runaway (TNR), the ejection and remnant hydrogen shell burning, and finally, the burned-out remnant left on the white dwarf. When more material is accreted from the secondary, the cycle starts over again. This simple drawing illustrates two of the assumptions used. First, materials from the three sources are thoroughly mixed by the TNR, and the mass of the remnant is the same as that from the previous outburst. These assumptions are supported by numerical simulations. Starrfield et al. (1972) found that the entire envelope above the TNR region is strongly convective and Shara, Prialnik, and Kovetz (1993) have shown that the remnant masses are very similar after the first few outbursts.

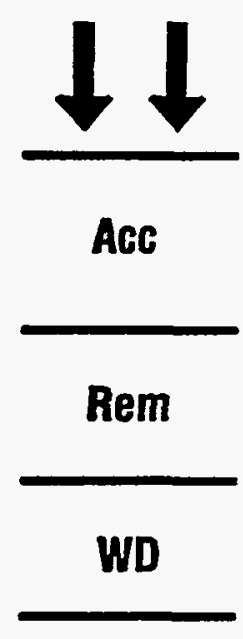

(a)

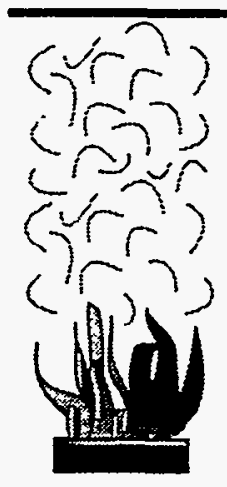

(b)
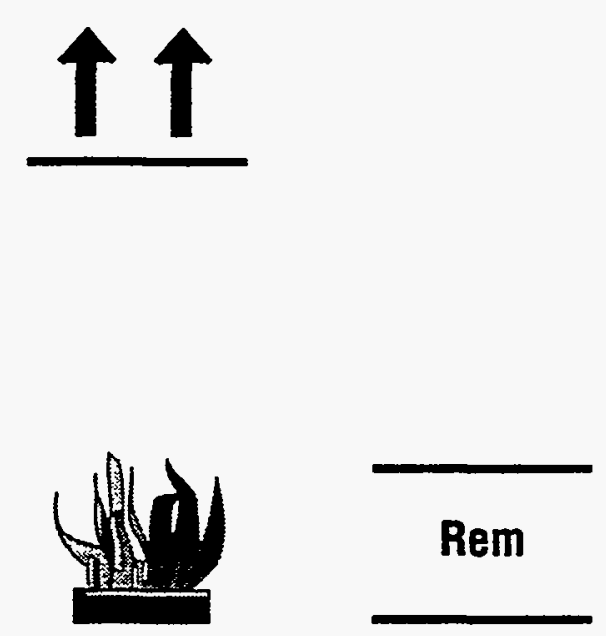

WD

(d)

Figure 1. The nova cycle showing (a) the accretion of material onto the white dwarf, (b) the mixing due to the TNR, (c) the ejection and remnant burning, and (d) the burned-out remnant. 


\section{Formulation of the Problem}

With the above assumptions, equating the mass abundances of hydrogen, helium, and the metals before and after the TNR produces the following equations:

$$
\begin{array}{r}
M_{a c c} \mathrm{X}_{0}=\mathrm{M}_{\text {tot }}\left(\mathrm{X}+\Delta \mathrm{X}_{\text {run }}\right) \\
\mathrm{M}_{a c c} \mathrm{Y}_{0}+\mathrm{M}_{\text {rem }}(\mathrm{X}+\mathrm{Y})=\mathrm{M}_{\text {tot }}\left(\mathrm{Y}-\Delta \mathrm{Y}_{\text {run }}\right) \\
\mathrm{M}_{\text {dg }}+\mathrm{M}_{\text {rem }} \mathrm{Z}+\mathrm{M}_{a c c} \mathrm{Z}_{0}=\mathrm{M}_{\text {tot }}\left(\mathrm{Z}-\Delta \mathrm{Z}_{\text {run }}\right) \\
\mathrm{M}_{\text {tot }}=\mathrm{M}_{a c c}+\mathrm{M}_{\text {rem }}+\mathrm{M}_{\text {dg }}
\end{array}
$$

where the abundances with a zero subscript and no subscript are for the secondary, and the mixed materials, respectively; $\mathrm{M}_{a c c}, \mathrm{M}_{\text {rem }}$, and $\mathrm{M}_{d g}$ are the accreted, remnant, and dredged-up white dwarf masses; and $\Delta \mathrm{X}_{\text {run }}$, $\Delta Y_{r u n}$, and $\Delta Z_{\text {run }}$ are the changes in the composition due to the TNR. The composition of the white dwarf is assumed to be all metals. This formulation is not new; variations of these equations have been described by Sparks et al. (1988), Prialnik (1990), and Fujimoto and Iben (1992). Note that any mixing mechanism before the TNR does not affect to this formulation.

Solving the above equations gives only a ratio of the individual mass components to the total mass. Another condition for one of these masses must be known in order to determine the individual mass components. The time for hydrogen burnout of the remnant mass provides such a condition. Equating the energy radiated from the remnant to the nuclear energy available from hydrogen burning gives

$$
\int \mathrm{L} d t=\mathrm{E}_{H} \mathrm{X} \mathrm{M}_{\mathrm{rem}}
$$

where $\mathrm{L}$ is the luminosity and $\mathrm{E}_{H}$ is the nuclear energy available from burning hydrogen to helium. We will assume that the luminosity is constant in time and equal to the equilibrium hydrogen shell burning plateau luminosity. Therefore,

$$
\mathrm{L}_{e q} \Delta \mathrm{t}=\mathrm{E}_{H} \mathrm{X} \mathrm{M}_{\text {rem }}
$$

\section{Results}

Let us now consider GQ Mus, a nova that erupted in 1983. Hassall et al. (1990) derived abundances of $\mathrm{X}=0.27, \mathrm{Y}=0.32$, and $\mathrm{Z}=0.41$ for its ejecta. It is assumed that the secondary has solar abundances $\left(\mathrm{X}_{0}=0.71\right.$, 
$Y_{0}=0.27$, and $Z_{0}=0.02$ ). We will further assume that the primary is a 1.0 $\mathrm{M}_{\odot} \mathrm{CO}$ white dwarf. The heavy element composition of the ejecta (Hassall et al., 1990) strongly suggests that the TNR occurred on a CO white dwarf, and $1.0 \mathrm{M}_{\odot}$ is a reasonable mass for such a white dwarf. Fortunately, the results depend only weakly on this chosen mass. For a $1.0 \mathrm{M}_{\odot}$ white dwarf, the amount of hydrogen burned during the TNR to produce enough energy to lift material to the white dwarf's Roche lobe radius (which for most nova binary systems is $\sim 1.0 R_{\odot}$ ) is 0.04 (see Fujimoto and Iben, 1992 for a more detailed discussion). For a TNR on a $\mathrm{CO}$ white dwarf, hydrogen is combined with $\mathrm{C}^{12}$ to produce mostly $\mathrm{C}^{13}$ and $\mathrm{N}^{14}$ (Starrfield, Truran, and Sparks, 1975). Thus, we will use $\Delta \mathrm{X}_{\text {run }}=\Delta \mathrm{Z}_{\text {run }}=0.04$ and $\Delta \mathrm{Y}_{\text {run }}=0.0$.

Substituting these values into eqs.(1-4) gives $M_{\text {rem }} / M_{\text {tot }}=0.34$, $\mathrm{M}_{\mathrm{acc}} / \mathrm{M}_{\text {tot }}=0.44$, and $\mathrm{M}_{d g} / \mathrm{M}_{\text {tot }}=0.22$. Since $\Delta \mathrm{X}_{\text {run }}$ and $\Delta \mathrm{Z}_{\text {run }}$ are small compared to the observed $\mathrm{X}(=0.27)$ and $\mathrm{Z}(=0.41)$ values, the assumed value of the white dwarf's mass has only a small effect on the final results. Recently, the X-rays from GQ Mus observed by ROSAT (Shanley et al., 1995) have shown a sharp decrease 11 years after outburst. They interpreted this decrease as the turn off of the remnant hydrogen shell source. Using the plateau luminosity of the appropiate hydrogen shell burning model of Fujimoto and Then (1992) in eq.(6), we calculate a remnant mass of $1.7 \times$ $10^{-5} \mathrm{M}_{\odot}$ for GQ Mus. This mass combined with the previous results yields an ejected mass of $3.3 \times 10^{-5} \mathrm{M}_{\odot}$ for GQ Mus.

One caveat to this simplified picture is that, according to Fujimoto and Iben (1992), the radius of this $1.7 \times 10^{-5} \mathrm{M}_{\odot}$ remnant will be much larger than any reasonable Roche lobe. In other words, there will be a common envelope around the binary, and the composition of the remnant may be a mixture from the ejecta and the secondary. As an extreme case, we can assume that the remnant material comes entirely from the secondary. Thus, the $\mathrm{X}$ in eq.(6) and the $\mathrm{X}, \mathrm{Y}$, and $\mathrm{Z}$ on the left hand side of eqs.(2) and (3) will have a zero subscript signifying material from the secondary. Under these conditions the remnant mass is $5.0 \times 10^{-6} \mathrm{M}_{\odot}$, and the ejected mass is $2.0 \times 10^{-5} \mathrm{M}_{\odot}$. A white dwarf remnant formed from a common envelope will lie somewhere between these two extreme cases, and thus the ejected mass lies between 2.0 and $3.3 \times 10^{-5} \mathrm{M}_{\odot}$.

V1974 Cyg is the only other nova observed by ROSAT to have a sharp decrease in X-rays. Its X-ray flux turned off 18 months after outburst (Krautter et al., 1995). Recent photoionization models of the emission lines by Austin et al. (1995) constrained the helium abundance from 0.22 to 0.46 and the metals from 0.36 to 0.44 . Since the emission lines indicate a large abundance of $\mathrm{O}, \mathrm{Ne}$, and $\mathrm{Mg}$, we will assume that the TNR occurred on a $1.25 \mathrm{M}_{\odot}$ ONeMg white dwarf. The amount of nuclear burning required to lift material off of a $1.25 \mathrm{M}_{\odot}$ white dwarf indicates that $\Delta \mathrm{X}_{\text {run }}=\Delta \mathrm{Y}_{\text {run }}$ 
$=0.08$ and $\Delta Z_{r u n}=0.0$ (see Fujimoto and Iben, 1992 and Politano et al., 1995). Here the assumed white dwarf's mass is more critical to the final results than in the case for GQ Mus. Using the largest values for both helium and the metals, we calculate a remnant mass of $8.7 \times 10^{-6}$ and 8.7 $\times 10^{-7} \mathrm{M}_{\odot}$ and an ejected mass of $7.1 \times 10^{-6}$ and $1.8 \times 10^{-6} \mathrm{M}_{\odot}$ when the composition of the remnant mass is like that of the ejected mass and the secondary, respectively. For the smallest deduced values of helium and metal abundances, the remnant mass is $2.1 \times 10^{-6}$ to $8.7 \times 10^{-7} \mathrm{M}_{\odot}$ for the same two sources of the remnant mass composition. However, since the lowest limit of the helium abundance can be produced during the TNR, no upper limit constraints can be placed on the ejected mass. Thus, we have found only a lower limit of $1.8 \times 10^{-6} \mathrm{M}_{\odot}$ for the ejected mass of V1974 Cyg.

\section{Conclusions}

This method uses the observed abundances from the ejecta and the turn-off time of the remnant burning to determine the ejected and remnant masses of a nova. At present only the rapid decrease in X-rays observed by ROSAT has been attributed to the remnant burnout and only for two novae: GQ Mus and V1974 Cyg. For GQ Mus, we find an ejected mass between 2.0 and $3.3 \times 10^{-5} \mathrm{M}_{\odot}$, whereas Hassall et al. (1990) estimated it to be 8.4 $\times 10^{-5} \mathrm{M}_{\odot}$. Stringfellow and Bowyer (1994) detected V1974 Cyg with the Extreme Ultraviolet Explorer $\sim 285$ days after its outburst. They fit their data best with a hot white dwarf atmosphere of $4.0 \pm 0.5 \times 10^{5} \mathrm{~K}$ and a column hydrogen density near $3 \times 10^{21} \mathrm{~cm}^{-2}$. These high temperatures require that the radius of the remnant on the white dwarf be small. For our assumed white dwarf mass of $1.25 \mathrm{M}_{\odot}$ and their temperatures, the unburnt remnant mass is estimated to be from 5.0 to $5.6 \times 10^{-7} \mathrm{M}_{\odot}$, using Fujimoto and Iben's (1992) graphs of equilibrium models. Since at the time of their observation, approximately one half of the remnant mass has burned out, this range of remnant masses is entirely consistent with our calculated range of $8.7 \times 10^{-7}$ to $2.1 \times 10^{-6} \mathrm{M}_{\odot}$. Krautter et al. (1995) calculated an upper limit of $2 \times 10^{-5} \mathrm{M}_{\odot}$ for the remnant mass of V1974 Cyg by equating its potential energy to the emitted energy from the latter part of the X-ray light curve. Although these various deduced masses for the remnant of V1974 Cyg are consistent, there is a large discrepancy in estimations of its ejected mass. We derive a lower limit of $1.8 \times 10^{-6} \mathrm{M}_{\odot}$ for the ejected mass in V1974 Cyg, whereas Shore et al. (1993) find a lower limit of $10^{-4} \mathrm{M}_{\odot}$. Future abundance or white dwarf mass determinations of V1974 Cyg can provide upper limits on its ejected mass with this new method. However, it is unlikely that the calculated ejected mass will be as 
large as $10^{-4} \mathrm{M}_{\odot}$. These ejected mass discrepancies must be resolved. If other criteria for the burnout of the remnant shell, for example, a rapid decrease of a high-ionization line, could be established, then this method could be applied to other novae.

\section{References}

Austin, S., Wagner, M., Starrfield, S., Shore, S., Sonneborn, G., and Burtran, R. 1995, these proceedings

Fujimoto, M. Y., and Iben, I. 1992, ApJ, 399, 646

Hassall, B. J. M., et al. 1990, in Physics of Classical Novae: IAU Colloquium No. 122 eds. A. Cassatella and R. Viotti (Berlin: Springer-Verlag), 202

Krautter, J., Ogelman, H., Starrfield, S., Wickmann, R., and Trumpter, J. 1995, in preparation

Politano, M., Starrfield, S., Truran, J. W., Weiss, A., and Sparks, W. M. 1995, to be published in $A p J$

Prialnik, D. 1990, in Physics of Classical Novae: IAU Colloquium No. 122 eds. A. Cassatella and $R$. Viotti (Berlin: Springer-Verlag), 351

Shanley, L., Ogelman, H., Gallagher, J. S., Orio, M., and Krautter, J. 1995, submitted to ApJ Letters

Shara, M. M., Prialnik, D., and Kovetz, A. 1993, ApJ, 406, 220

Shore, S. N., Sonneborn, G., Starrfield, S., Gonzalez-Riestra, R., and Ake, T. B. 1993, AJ, 106, 2408

Sparks, W. M., Starrfield, S. G., Truran, J. W., and Kutter, G. S. 1988, in Atmospheric Diagnostics of Stellar Evolution: Chemical Peculiarity, Mass Loss and Explosion: IAU Colloquium No. 108 ed. K. Nomoto (Berlin: Springer-Verlag)

Starrfield, S., Truran, J. W., and Sparks, W. M. 1975, ApJ, 198, L113

Starrfield, S., Truran, J. W., Sparks, W. M., and Kutter, G. S. 1972, ApJ, 176, 169

Stringfellow, G. S., and Bowyer, S. 1994, in Interacting Binary Stars ed. A. W. Shafter (San Francisco: ASP), 315

\section{DISCLAIMER}

This report was prepared as an account of work sponsored by an agency of the United States Government. Neither the United States Government nor any agency thereof, nor any of their Government. Neithes the Unity, express or implied, or assumes any legal liability or responsiemployees, makes any warranty, express or implied, or assuness information, apparatus, product, or bility for the accuracy, completeness, or usefuld not infringe privately owned rights. Referprocess disclosed, or represents that its use would not in service by trade name, trademark, ence herein to any specific commercial product, process, or ser imply its endorsement, recommanufacturer, or otherwise does not necessarily constite or inply its ency thereof. The views mendation, or favoring by the United States Government or any agency the necessarily state or reflect those of the

and opinions of authors expressed herein do not 